\title{
PROKLA-Redaktion
}

\section{Editorial: Illusion und Macht des Geldes}

In Krisensituationen wird der gesellschaftliche Umgang mit Geld verstärkt zum Problem. So auch in der großen Krise, die sich seit 2007 ausgehend von den USA entwickelt hat und heute vor allem als Griechenland- und Eurokrise die öffentliche Diskussion bestimmt. Als die ersten Krisensymptome sich 2007 bemerkbar machten, stand die Geldpolitik im Zentrum der Bemühungen, die Krise einzudämmen. Der US-amerikanische Federal Reserve Board, die Europäische Zentralbank (EZB) und andere wichtige Zentralbanken schlossen Swap-Abkommen zur gegenseitigen Unterstützung ab, senkten die Zinsen praktisch auf null und pumpten in koordinierten Aktionen verstärkt Geld in das Bankensystem, um dem Zusammenbruch des Geldmarktes und des gesamten Finanzsystems in der westlichen Welt entgegenzuwirken. In den folgenden Jahren kam es zu einer sukzessiven Lockerung der Geldpolitik, die sich in einem drastischen Anstieg der Bilanzsumme der führenden Zentralbanken ausdrückte. Banken wurde nahezu unbegrenzt Geld zur Verfügung gestellt. Die Krise konnte durch die Geldpolitik alleine jedoch nicht überwunden werden. Bis heute ist die globale Konjunktur so labil, dass die führenden Zentralbanken vor einer Anhebung der Zinsen zurückschrecken, die aus der gegenwärtigen Stagnation eine erneute Rezession und Deflation machen könnte.

Die Lockerung der Geldpolitik in der Krise ist keineswegs nur ein keynesianisches
Konzept. Vielmehr sahen auch modernere Mainstream-Ökonomen (manchmal NeuKeynesianer genannt) wie Ben Bernanke, der von 2006 bis 2014 Präsident des Federal Reserve Board war, darin das Mittel der Wahl, um die Krise zu bekämpfen. Nahezu völlig irrelevant für Zentralbanken wurde der hergebrachte Monetarismus, der eine exogene Steuerung der Geldmenge anstrebt und einen engen Zusammenhang zwischen Geldmenge und Inflation postuliert. Der Unterschied zwischen modernen Mainstream-Ökonomen und traditionellen Keynesianern besteht allerdings darin, dass erstere in der Geldpolitik gleichsam ein Allheilmittel sehen, während Keynesianer - zu Recht - auf ihre Grenzen hinweisen und auch für fiskalpolitische Maßnahmen plädieren, um der Krise entgegenzuwirken. Aus marxistischer Perspektive überschätzen freilich beide Schulen die Möglichkeiten wirtschaftspolitischer Maßnahmen.

Die Lockerung der Geldpolitik ist nicht unumstritten, wobei die Fronten quer durch die politischen Lager verlaufen. Unter den Neoliberalen, die dem traditionellen Monetarismus anhängen und in der Deutschen Bundesbank eine Trutzburg gefunden haben, sind es die Anhänger der österreichischen Schule, die die Geldschwemme der Zentralbanken für gefährlich halten und in einer zu lockeren Geldpolitik sogar die zentrale Krisenursache sehen. So wärmt etwa die Frankfurter Allgemeine Zeitung die Krisendiagnosen 
von Hayek und Mises beinahe täglich neu auf. Linke Kritiker thematisieren dagegen vor allem die sozialen Folgen der lockeren Geldpolitik, insbesondere die Beförderung einer neuen Blase auf den Aktienmärkten und die zunehmend ungleiche Verteilung von Vermögen und Einkommen, die dadurch beschleunigt wird, dass das zusätzliche Geld jenen zufließt, die sowieso bereits reichlich darüber verfügen (vgl. dazu den Beitrag von Maria Kader und Uli Schwarzer in diesem Heft).

Kontrovers ist nicht nur, wie viel Geld in den Wirtschaftskreislauf gepumpt, sondern auch, wie die gesellschaftliche Versorgung mit Geld überhaupt geregelt werden sollte. Nachdem die globale Finanzkrise sich im Herbst 2008 zugespitzt hatte, wurden in den USA, in der EU und in der G 20 zahlreiche Politiken zur Reform des Kreditwesens diskutiert. Christoph Scherrer macht in seiner Analyse der entsprechenden Maßnahmen klar, dass die damals debattierten Maßnahmen nur zum Teil umgesetzt wurden und dass die Reformen den Handlungsspielraum der Banken und vor allem der Nicht-Bank-Finanzintermediäre nicht wesentlich eingeschränkt haben. Das Finanzkapital sei trotz bzw. wegen der Reformen weiterhin hegemonial.

Die beschränkte Reformagenda der herrschenden Kräfte hat von Anfang viele Kritiker auf den Plan gerufen, die weitreichendere Veränderungen der Regulation des Geldes und des Kredits forderten. Es gibt eine Vielfalt an Reformvorschlägen und alternativen Praktiken des Umgangs mit Geld, die mehr oder minder weitreichend sind. Beat Weber setzt sich in seinem Beitrag kritisch mit den Konzepten des Vollgeldes und des Regionalgeldes, mit den Reformvorschlägen der Modern Monetary Theory und mit Bitcoin als privat geschaffenem, elektronischem Geld auseinander. Derartige Geldreformvorschläge werden längst nicht mehr nur von relativ randständigen Akteuren verfochten. Inzwischen prüft z.B. die Regierung Islands einen Vorschlag zur Einführung von Vollgeld. Und Peter Bofinger, Mitglied im Sachverständigenrat der Bundesregierung zur Begutachtung der gesamtwirtschaftlichen Entwicklung, fordert die Abschaffung des Bargeldes zugunsten des elektronischen Geldes, um die staatliche Fähigkeit, die Wirtschaft zu kontrollieren, zu erhöhen.

Vielen dieser Geldreformkonzepte ist bei allen sonstigen Unterschieden gemein, dass sie die Wurzeln der kapitalistischen Krisen in der herrschenden Form des Geldes sehen und diese einer Kritik unterziehen, ohne ihren Zusammenhang mit der kapitalistischen Produktionsweise als solcher zu thematisieren. Das Geld soll reformiert werden, ohne die Warenproduktion anzutasten. Wie in der neoklassischen Theorie bleibt Geld ein Störfaktor eines an sich stabilen ökonomischen Systems (siehe dazu den Beitrag von Ingo Stützle). Dabei bleibt häufig unklar, was Geld überhaupt ist. Es wird oft nicht klar zwischen der Geldform als solcher und den verschiedenen Formen des Geldes, zwischen Geld, Kredit, Kreditgeld, Zentralbankgeld, Giralgeld usw. unterschieden. Wird die Geldfrage nicht im Rahmen einer kapitalismuskritischen Perspektive eingebettet, so werden viele Fragen gar nicht gestellt und viele Voraussetzungen der herrschenden Lehre reproduziert - trotz der Sehnsucht nach heilen Zuständen.

Aber auch die radikale, antikapitalistische Linke tut sich schwer mit Geld und Kredit als Gegenstand der Kritik und der politischen Intervention. Dies wurde z.B. bei der Blockupy-Mobilisierung deutlich, als viele zweifelten, ob die Bankenmetropole und die EZB der richtige Ort für den Protest seien. Die radikale Linke hatte sich offensichtlich kaum damit beschäftigt, was 
die EZB macht, wie das Geld in die Welt kommt oder was Geldpolitik bedeutet. Dabei sind Zentralbanken und ihre Geldpolitik eine Grundvoraussetzung für die Reproduktion des modernen Kapitalismus. Auch Kräfte, die über den Kapitalismus hinausgehen wollen, sollten sich mit der Regulation von Geld und Kredit beschäftigen. Dass Zentralbanken einer demokratischen Kontrolle unterworfen werden müssen, zeigt sich aktuell gerade im Verhältnis der EZB zu Griechenland. Die EZB hat den griechischen Banken den Geldhahn bewusst ein Stück weit zugedreht und versucht damit, die griechische Regierung zu erpressen und sie zur Fortsetzung der Austeritätspolitik zu zwingen, ohne dass dies in der breiten europäischen Öffentlichkeit überhaupt angemessen wahrgenommen wird. Mainstream-Medien gefallen sich weiterhin darin, die Stereotype „Verträge muss man einhalten“ und „Die Griechen haben über ihre Verhältnisse gelebt" unermüdlich zu wiederholen. Dabei wäre es die Pflicht der EZB, für die griechischen Banken weiterhin als lender of last resort zu fungieren. Stattdessen droht sie unverhohlen damit, im Falle eines Staatsbankrotts die griechischen Banken ganz von der Refinanzierung abzuschneiden und so Griechenland aus der Eurozone hinauszuwerfen, obwohl es dafür keine rechtliche Grundlage gibt. Die vermeintlich politisch neutrale Geldpolitik der EZB wird so zur zentralen Waffe der Herrschenden im Kampf gegen eine Regierung, die dabei ist, eine Alternative zum Neoliberalismus praktisch durchzusetzen (siehe dazu den Beitrag von Martin Konecny).

An Literatur zum Thema Geld mangelt es nicht, ja es gibt - nicht zuletzt wegen der jüngsten Finanz- und Wirtschaftskrise - geradezu einen Boom. Die Autorinnen und Autoren kommen aus den unterschiedlichsten Richtungen, erwähnt seien z.B. die Bücher von Christina von Braun, Christian Felber, Niall Ferguson, David Graeber, Ulrike Herrmann, Joseph Huber, Felix Martin, Christoph Türcke und Joseph Vogl. Bei der Suche nach dem "Ursprung" des Geldes gehen viele - wie etwa Graeber - historisch vor. Sie suchen Antworten auf das "Geldrätsel " in der Vergangenheit, übertreffen sich darin, wie weit sie zurückgehen und widersprechen sich in der Frage, was das durch die Zeit tragende Charakteristikum ist. In systematischer Hinsicht fallen mehrere neuere Analysen allerdings weit hinter die marxsche Geldtheorie zurück. Ingo Stützle und Heiner Ganßmann versuchen mit ihren Beiträgen, Licht in die heillose Verwirrung zu bringen, die die gegenwärtigen politischen und ökonomischen Diskussionen über Geld prägt. Eine Systematisierung der Geldund Kredittheorie ist dringend notwendig. Die Systematisierung muss aber auch die historischen Verschiebungen in den Geldverhältnissen umfassen, die sich im Laufe der kapitalistischen Entwicklung ergeben haben - etwa in der Frage, ob Geld noch an eine Ware wie Gold rückgekoppelt ist und was als Weltgeld fungiert. Waren dies früher die Geldware Gold und das britische Pfund Sterling, so ist es heute vor allem der US-Dollar. Doch dieser ist nicht unangefochten. Hansjörg Herr geht in seinem Beitrag der Entwicklung der Währungskonkurrenz unter den Bedingungen der neoliberalen Globalisierung nach.

Die verschiedenen Beiträge in diesem Heft machen auch deutlich, dass selbst Autoren, die auf den ersten Blick politisch und theoretisch nicht so weit auseinander zu stehen scheinen, doch zu recht unterschiedlichen Einschätzungen gelangen können, wenn es um die Beurteilung der Entwicklungsdynamik des gegenwärtigen Kapitalismus und der herrschenden Krisenpolitik geht. Dem liegen nicht zuletzt 
auch unterschiedliche Sichtweisen des Zusammenhangs von Geld, Kredit und Finanzmärkten zugrunde, wie Michael $W e n d l$ in seiner Kritik der Arbeiten von Jörg Huffschmid und Stephan Krüger deutlich macht. So baut Wendl beispielsweise wie Huffschmid auf den Theorien von Marx und Keynes auf, weist aber Huffschmids These einer Dominanz der Finanzmärkte im gegenwärtigen Kapitalismus zurück.

Wendls Kritik erinnert an frühere Diskussionen über Veränderungen der Entwicklungsweise des Kapitalismus und die Entkopplung von „realer" und „monetärer" Akkumulation, die auch in der PROKLA geführt wurden (und unter prokla.de frei zugänglich im Archiv zu finden sind). Verwiesen sei etwa auf die Beiträge Die Transformation des Finanzkapitals von Robert Guttmann und Money Makes the World Go Round. Über die Verselbständigung der Geldsphäre und andere Mißverständnisse von Michael Heine und Hansjörg Herr in PROKLA 103 (1996). Überhaupt lohnt es sich, dieses Heft sowie PROKLA 63 (1986), die beiden früheren Hefte zum Thema Geld, wieder zu lesen. Trotz veränderter historischer Bedingungen bleiben viele Probleme, die damals schon diskutiert wurden, aktuell. Ist es heute die globale Finanz- und Wirtschaftskrise seit 2008, die dazu zwingt, über die Hegemonie des
Finanzkapitals und die Gläubiger-Schuldner-Beziehungen nachzudenken, so waren es 1986 die lateinamerikanische Schuldenkrise und 1996 die neuen Bedingungen der Globalisierung. Hansjörg Herr schlug sich schon 1986 mit dem verblichenen Silvio Gesell herum, der mit seinen Thesen heute noch oder wieder bei etlichen Geldreformern Pate steht. Elmar Altvater und Raul Rojas beschäftigten sich bereits 1996 mit elektronischem Geld.

Wir freuen uns, dass wir mit Heiner Ganßmann und Hansjörg Herr zwei ehemalige Redaktionsmitglieder und Autoren der früheren Geld-Hefte auch für Beiträge in dem vorliegenden Heft gewinnen konnten. Hansjörg Herr hat darüber hinaus als Gastredakteur an diesem Heft mitgewirkt, wofür die Redaktion ihm ganz herzlich dankt.

Außerhalb des Schwerpunkts publizieren wir hier neben dem Beitrag von Martin Konecny zur Entwicklung in Griechenland noch eine Analyse von Fatma Umul zu den diskursiven Veränderungen in der türkischen Frauenpolitik nach dem Gezi-Aufstand. Mit letzterem hatte sich in PROKLA 173 (2013) bereits Errol Babacan auseinandergesetzt.

\author{
Hansjörg Herr, Thomas Sablowski \\ und Ingo Stützle \\ (Für die Redaktion)
}

